
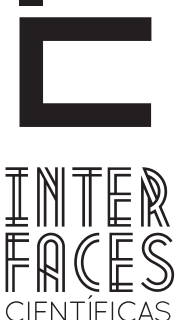

HUMANASE SOCIAIS

\title{
AVALIAÇ̃̃o DE PROGRAMA DE CAPACITAÇ̃̃ PARA SUA INCLUSÃO DIGITAL POR AGENTES COMUNITÁRIOS DE SAÚDE
}

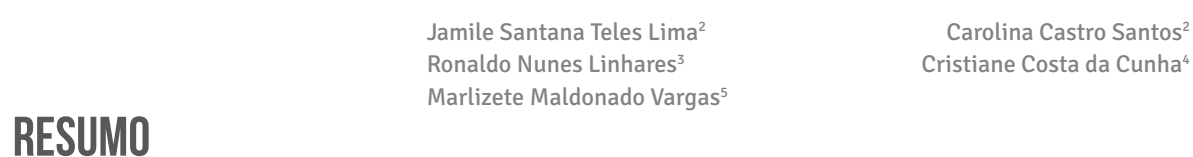

Projetos do Núcleo de Estudos em Saúde Pública/SE capacitam Agentes Comunitários de Saúde (ACS) com o propósito de habilitá-los para o manuseio de recursos digitais, acesso aos meios de comunicação online e participarem de cursos de educação à distância. Este estudo identificou forças e fraquezas do projeto desenvolvido em Sergipe em parceria com a UnB. Analisou opiniões e percepções dos ACS acerca das contribuições desta capacitação para suas ações quotidianas. Através de levantamento com uma amostra de 112 sujeitos, identificou-se que $70 \%$ dos ACS não possuíam recursos multimídia; $63 \%$ afirmaram que os conteúdos foram acessíveis e $30 \%$ consideraram que a capacitação iria contribuir para seu trabalho. Dados levantados através de Grupos Focais destacaram dificuldades de gerenciamento do projeto: estrutura física, relacionamento interpessoal com monitores, desnivelamento entre capacitados, material didático subutilizado. Dentre as forças: entrosamento entre participantes, possibilidade de maior qualificação. Conclui que projetos relevantes como esse necessitam de preparação dos gestores locais e adequação às condições dos usuários que aperfeiçoem os benefícios.

\section{PALAVRAS-CHAVE}

Agentes Comunitários de Saúde. Inclusão Digital. Avaliação 


\section{ABSTRACT}

Projects of the Center for Studies in Public Health/SE enable the Community Health Agents ( $\mathrm{CHA}$ ) in order to enable them to handle digital resources, access to online media and participate in distance education courses. This study identified strengths and weaknesses of the project in Sergipe developed in partnership with UNB. Examined the perceptions and opinions about the $\mathrm{CHA}$ contributions of this training to their everyday actions. Through a survey with a sample of 112 subjects, it was found that $70 \%$ of $\mathrm{CHA}$ did not have multimedia features, $63 \%$ stated that the contents were accessible and $30 \%$ felt that the training would contribute to their work. Data collected through focus groups highlighted difficulties in project management: physical structure, interpersonal relationship with monitors, unevenness between trained, courseware underutilized. Among the forces: understanding between participants, possibility of higher qualification. Concludes that relevant projects like this require preparation of local managers and adaptation to conditions that enhance users' benefits.

\section{KEYWORDS}

Community Health Workers. Digital Inclusion. Evaluation.

\section{INTRODUÇ̄̃̃O}

Para uma compreensão menos simplificada de promoção de saúde é necessário intensificar as ações e estratégias de promoção no cotidiano dos serviços de saúde e promover a autonomia dos indivíduos e profissionais. Uma das formas é a capacitação continuada dos mesmos (HEIDMANN, 2006).

\section{RESUMEN}

Proyectos del Centro de Estudios en Salud Pública / SE capacitan Agentes Comunitarios de Salud (ACS) con el fin de que puedan manejar los recursos digitales, accesar a los medios de comunicación en línea y realizar clases a distancia. Este estudio identificó las fortalezas y debilidades del proyecto desarrollado en Sergipe/Brasil, en colaboración con la Universidad de Brasilia. Fueron analizadas opiniones y percepciones de los ACS acerca de las contribuciones de este entrenamiento para sus actividades cotididianas. Se hizo una recolección de datos con una muestra de 112 sujetos que apuntó que $70 \%$ de los ACS no tenian recursos multimedia; para el $63 \%$ de los entrevistados los contenidos eran accesibles y el $30 \%$ consideró que la capacitación podría aportar en su trabajo. Los datos recogidos a través de grupos focales destacaron las dificultades de la gestión del proyecto: estructura física, las relaciones interpersonales con los monitores, desigualdad técnica entre los entrenados , material didáctico subutilizado. Entre los puntos positivos tuvieron destaque: la relación entre los participantes y la posibilidad de una calificación más alta. Se concluye que este tipo de proyecto requiere preparo de los gestores locales y adaptación a las condiciones de los usuarios que perfeccionen los beneficios.

\section{PALABRAS CLAVE}

Agentes Comunitarios de Salud. Inclusión digital. Evaluación

O Programa de Agentes Comunitários de Saúde (PACS), criado em junho de 1991, pelo Ministério da Saúde (2002), fundamenta-se na compreensão da importância da saúde como um direito e a educação e a comunicação enquanto situação de perfeito bem-estar físico, mental e social, que está atrelado às 
condições de vida dos sujeitos, grupos e populações e a construção de uma cidadania plena.

Lévy (2003) afirma que se deve buscar na técnica, potenciais subjetivos e cognitivos, e com apropriação da cultura tecnológica para a expansão do pensamento, conhecimento e das relações interpessoais, através de tecnologias inteligentes que ampliam as possibilidades de construção de redes colaborativas e interativas de construção do conhecimento. Sendo assim, o meio digital possibilita potencializar o desenvolvimento humano saudável. E o processo de infoinclusão social deve acrescentar ao mundo digital um novo espaço de socialização, pesquisa e aprendizagem.

Segundo o Instituto Brasileiro de Geografia e Estatística (IBGE, 2000), nos últimos cinco anos, houve uma forte aceleração na inclusão digital no Brasil. Pesquisas revelaram que o Brasil é o quinto país com maior número de conexões de internet e que 67,5 milhões de pessoas acessaram a Internet (IBOPE, 2009).

Tanto no campo da educação como no do trabalho, as Tecnologias de Informação e Comunicação (TICs) podem facilitar o trabalho de entendimento dos conceitos e estratégias utilizados e superar as dificuldades de acesso às informações, facilitando a construção de novos conhecimentos. A educação voltada aos meios tecnológicos visa a apropriação coletiva do conhecimento, um saber interativo. 0 uso das novas TICs na educação torna-se uma matriz que transforma o aprendizado por meio de conteúdos transmitidos através de recursos interativos (CARAM; BIZELLI, 2011).

Para que a inclusão digital seja funcional é preciso que ela garanta a acessibilidade e conectividade à sociedade de informação e "que proporcione melhores condições de vida e uma cidadania local possível” (GUERREIRO, 2006, p. 174). Dessa forma, a infoinclusão não é apenas a aquisição de habilidades e de domínio técnico, mas sim possibilita o fortalecimento do cidadão, para o agir comunicativo, consciente, através de práticas de educação emancipatória (FREIRE, 1979, citado por PAIXÃO; FREIRE; LIMA; LINHARES, 2010).

As transformações possíveis advindas de uma política pública de saúde têm na educação e nos processos de comunicações sociais, parceiros fundamentais. $E$, nas tecnologias, mediadores eficientes. 0 conceito de aprendizagem digital é amplamente utilizado por diversos modelos de ensino à distância, essas metodologias podem oferecer bases de qualidade para o processo de inclusão digital, sendo que essa aprendizagem ocorre no próprio local de trabalho com a finalidade de capacitar os profissionais (DIAS, 2000).

A partir da ideia e fomentar o trabalho em promoção da saúde, foi desenvolvido em Sergipe o Projeto denominado Inclusão Digital de Agentes Comunitários de Saúde. Articulado com as Unidades de Estudos e Pesquisas em Saúde da Família (UEPSF) e de Tecnologias da Informação e Comunicação em Saúde (UTICS) do Núcleo de Estudos em Saúde Pública (NESP) da Universidade de Brasília (UnB) e a parceria com o Núcleo de Pós-Graduação em Educação da Universidade Tiradentes e Secretarias Municipais de Saúde. Executado em duas etapas: oficina de introdução à informática, e oficinas online com temáticas específicas para pratica profissional dos ACS.

Neste sentido, a estratégia de educação a distancia mediada pelas TIC permite ao profissional de saúde e em especial aos ACS, um ambiente virtual de aprendizagem que amplia suas possibilidades de formação continuada e o desenvolvimento de competências e habilidades fundamentais para uma aprendizagem autônoma, interativa, colaborativa e de pesquisa (VALENTE et. al., 2005).

A $1^{\mathrm{a}}$ etapa objetivava o desenvolvimento de ações como: criação de laboratórios de formação e instrumentalização do agente de saúde para sua atuação na mobilização da participação popular; a aquisição 
de competências e habilidades infosociais que contribuam para a cidadania plena. Já a segunda garantiria acesso à informação e conhecimento atualizado, assim como a construção de novos conhecimentos advindos das práxis desses profissionais, disponibilizadas em rede. Pretendia-se também que tais ações levassem à maior participação e empoderamento dos ACS sobre educação em saúde através das TIC`s.

Esta pesquisa se propôs a verificar os resultados e impactos da capacitação para utilização de recursos

\section{MÉTODO}

Trata-se de uma pesquisa múltimétodo (PMM), com levantamentos tipo survey e por meio de grupos focais com análises das percepções e atitudes de usuários de projeto de inclusão digital.

O presente artigo é inédito e foi aprovado pelo Comitê de Ética em Pesquisa (CEP) pela Universidade Tiradentes, cujo protocolo é 050709. Esse teve ainda o apoio do Programa de Iniciação Científica da Universidade Tiradentes (PROBIC).

Os locais da pesquisa foram os Núcleos de Apoio a Tecnologia em Saúde incluídos no Projeto, que é desenvolvido com a UNB e o Núcleo de Pós Graduação em Educação da UNIT, com financiamento do Ministério da Ciência e Tecnologia, são eles: Itabaiana, Propriá e Lagarto.

Foram sujeitos 112 Agentes Comunitários de Saúde capacitados pelo programa de inclusão digital (amos- multimídias e educação à distancia de Agentes Comunitários de Saúde, bem como identificar, discutir e analisar opiniões, percepções, sentimentos e atitudes dos ACS acerca das contribuições da capacitação para suas ações quotidianas e discutir acerca das forças e fraquezas do programa de inclusão digital que se utiliza da EAD como método de disseminação de informação, comunicação e educação em saúde. tra obtida pela fórmula de Barbetta), nos polos dos municípios sergipanos de Itabaiana, Lagarto e Propriá.

Para levantamento dos dados quantitativos utilizou-se um formulário composto por 18 questões - 16 fechadas e 2 abertas, contendo variáveis que contemplavam desde o espaço físico até o relacionamento interpessoal do grupo e deste com os monitores. Para a análise desses dados foi utilizado o software SPSS 16.0, para levantamento de frequências relativas, médias, testes de análise de consistência de dados entre os grupos através do teste do qui - quadrado, considerando a margem de erro de $0,05 \%$.

Para o estudo qualitativo das percepções e sentimentos dos ACS acerca da capacitação, foram realizados Grupos Focais, que se apóiam na interação entre seus participantes para colher dados a partir desses temas disparadores.

\section{RESULTADOS}

0 perfil sociodemográfico dos participantes em relação à média das idades foi de 36 anos (variando de 22 a 62 anos), $75 \%$ finalizaram o ensino médio e $25 \%$ o ensino superior. Quanto à situação de renda, 
29\% recebem um salário mínimo; $68 \%$ um salário e meio; $2 \%$ dois salários e apenas $1 \%$ recebem 4 salários mínimos. Em relação ao tipo de moradia vivem em unidades e $77 \%$ unifamiliares e $23 \%$, em multifamiliares, sendo que $79 \%$ residem em casa própria, $19 \%$ pagam aluguel e $2 \%$ em invasões.

Constatou-se que a respostas dos ACS apontam que os conteúdos da capacitação foram de média relevância e que complementam conhecimentos adquiridos antes da oficina de inclusão digital, principalmente em relação à acessibilidade dos conteúdos, assim como a leitura dos textos oferecidos serem de fácil compreensão (Tabela 1). Destaca-se que na realização do grupo focal, muitos ACS relataram como dificuldade da capacitação o uso de apostilas continuadas, uma vez que essa prática facilitaria o aprendizado sobre a temática.

TABELA - Avaliação pelos ACS dos conteúdos e metodologia do projeto

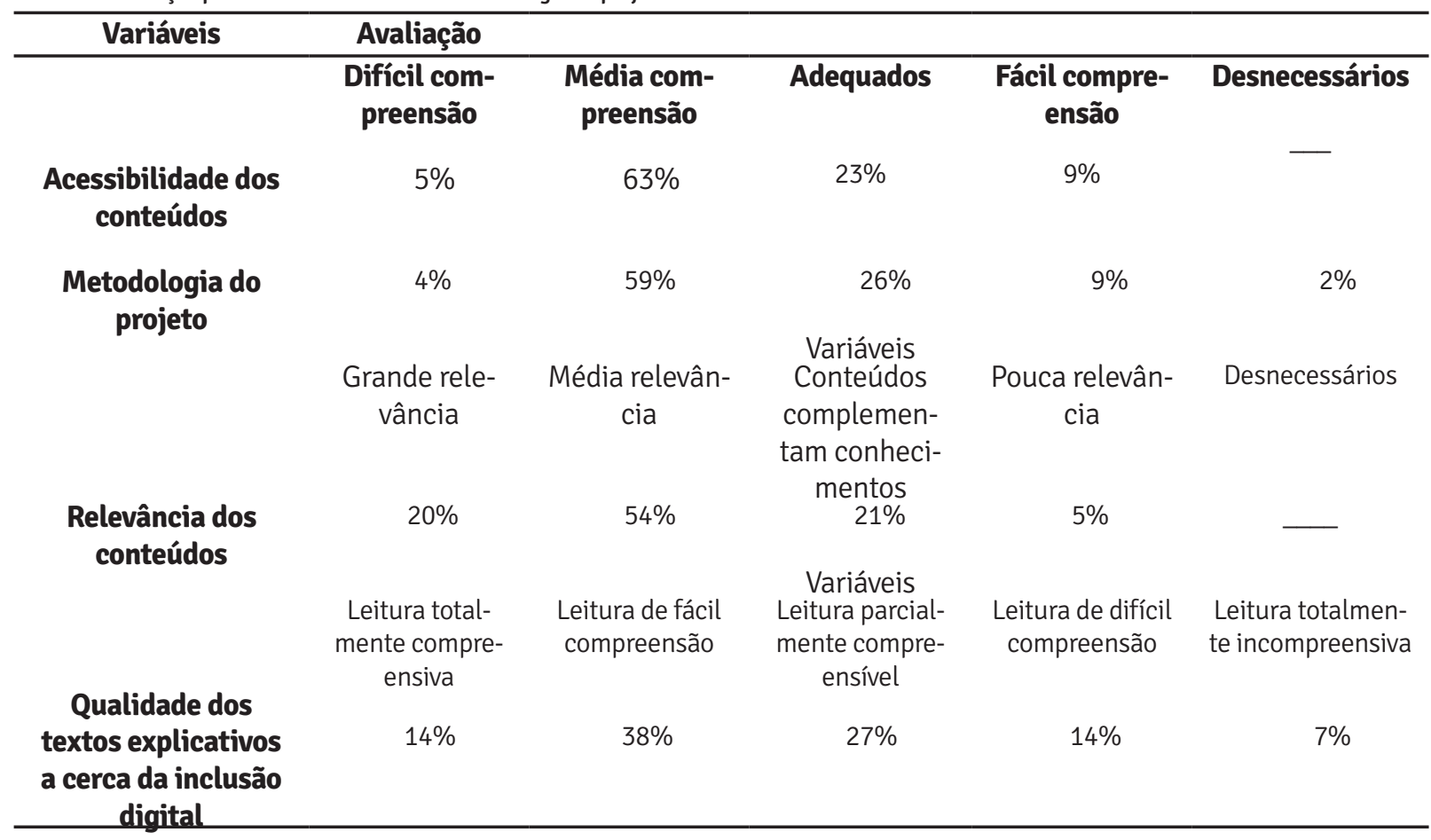

Fonte: Elaborado pelos autores

Quanto à estrutura física dos laboratórios de informática, 34\% dos sujeitos consideraram inadequadas. 0 acesso aos equipamentos e internet foi permitido, mas não atendeu às necessidades de $62 \%$ dos ACS. No grupo focal, os ACS relataram que o laboratório ficava fechado, uma vez que para esse ficar aberto era necessário um monitor diário, ou ainda que o labora- tório foi desfeito depois da capacitação inicial e que alguns computadores estavam nas secretarias dos municípios.

Quanto à relação aluno/computador, a média foi de dois para um. Pesquisas apontam como dificuldade de uma capacitação quando um computador é usado 
por mais de três pessoas, já que o tempo em que cada indivíduo pratica diminui consideravelmente (VALENTE et. al., 2005). Merece destaque ainda que $70 \%$ dos ACS não possuíam recursos multimídia em casa, o que contribui para o analfabetismo digital entre os participantes, além de ter sido um dos fatores que cooperou para que esses tivessem dificuldade em aprender o conteúdo transmitido. Segundo dado do Comitê Gestor da internet no Brasil (2006) o percentual de residências com computadores atinge em torno apenas $19,6 \%$.

No que se refere à contribuição da capacitação para qualificação do seu trabalho, $31 \%$ responderam que a capacitação foi suficiente para contribuir na qualidade do atendimento da população. No entanto foi possível detectar, na análise qualitativa, que os ACS tinham muita expectativa com a capacitação, mas diante as várias dificuldades enfrentadas foram se desmotivando e o impacto na qualificação de seu trabalho não foi percebido.

Os dados referentes às facilidades e dificuldades das relações interpessoais entre os ACS durante a implantação e desenvolvimento do projeto, assim como possibilidade de sugestões para melhoria da capacitação, por implicarem em respostas subjetivas, foram analisadas quanto ao conteúdo, e agrupadas em categorias conforme o Quadro 1.

Quadro1 - Contribuição do programa de inclusão digital para suas ações quotidianas, segundo os ACS capacitados nos pólos de Sergipe, 2011

\begin{tabular}{lc}
\multicolumn{1}{c}{ Categorias } & Freq. de respostas \\
\hline Juízos valorativos & 56 \\
Positivos & 45 \\
Negativos & \\
\hline Significados da capacitação & 39 \\
Promoveu trocas de experiências & 37 \\
Promoveu aperfeiçoamento no manejo com o usuário & 35 \\
Formou grupos homogêneos & 22 \\
Proximidade com os colegas & 17 \\
Aprendizagem de recursos multimídias & \\
\hline Dificuldades Percebidas & 54 \\
Dificuldade em entender as instruções do monitor & 37 \\
Dificuldade na compreensão dos conteúdos teóricos & 28 \\
Falta/falhas de comunicação & 10 \\
Não Houve & \\
\hline
\end{tabular}

\section{Fonte: Elaborado pelos autores}

Foram identificadas algumas dificuldades e facilidades que concorreram para o aproveitamento ou não da capacitação pelos ACS. Dentre as dificuldades os ACS de Itabaiana destacaram como queixa principal o relacionamento interpessoal com as duas monitoras, essas não teriam postura didática, o que teria dificultado na aprendizagem dos participantes. As facilitadoras alegaram não estarem motivadas em ensinar porque seus salários estarem atrasados.
Já, os ACS de Propriá destacaram como dificuldade principal o nivelamento entre os participantes, pois alguns já tinham um embasamento teórico e manejo com computador, enquanto outros sequer tinham, ao menos, ligado um computador. Nesse sentido houve certo prejuízo na aprendizagem, conforme exemplifica a seguinte fala: "Porque assim, eu que já sabia um pouco de computador tinha que esperar os que não sabiam mexer com computador aprender, no final das contas eu acabei até desaprendendo" (S8). 
Como dificuldade secundária os ACS destacaram que a página da plataforma era bastante complicada, com muitos atalhos, por isso os participantes alegaram não conseguir acessá-la, muito menos conectar-se com a finalização da capacitação.

Os Agentes Comunitários de Saúde de Lagarto enfatizaram como dificuldade principal a quantidade de aulas teóricas na capacitação e a ausência de material didático, como apostilas, que podiam facilitar a aprendizagem sobre os recursos multimídias. E como dificuldade secundária, a sala de laboratório que foi desativada após a capacitação de inclusão digital. Os participantes questionaram enfaticamente essa desativação, já que não era algo previsto pelo projeto e que contribuiria para reforçar toda aprendizagem, uma vez que teriam um espaço especializado para treinar o manejo com os recursos tecnológicos.

Pode-se destacar como dificuldades em comum aos ACS analisados nessa pesquisa: maior duração da capacitação, a estrutura física dos laboratórios (pequenos, sem ventilação e iluminação adequada), uma vez que apenas o município de Itabaiana estruturou um laboratório intitulado por Centro de Apoio Tecnológico para Inclusão Digital (CATIS), destacaram que o acesso a internet era restrito, o fato de não ser um computador para cada um dos agentes, além da capacitação usar o programa Linux, pois, segundo os agentes esse programa é muito complicado e de difícil manejo. Pode-se destacar que os ACS não estavam habituados a utilizar esse programa em suas vivências.

Em relação às forças da capacitação em inclusão digital, os ACS identificaram a melhora no relacionamento interpessoal entre os participantes, por meio da interação durante a capacitação, que por sua vez estimulou a troca de experiências. Além de contribuir para a aprendizagem em pesquisas sobre algumas doenças, o que favoreceu o repasse de conhecimentos adquiridos por meio dos recursos tecnológicos, sendo reforçado pelas práticas educativas na comunidade. Exemplificado pela fala dos Sujeitos 17 e 24:
0 bom é que com a internet a gente pode pesquisar sobre temas e tirar dúvidas da comunidade (S24).

Aprendi a organizar meu trabalho, troquei as fichas de papel, pelas tabelas do Excel (S17).

Os participantes dos pólos supracitados enfatizaram a importância de sua função para a comunidade, por serem o elo comunidade-serviço de saúde, multiplicadores de informação, educadores na prática preventiva sobre epidemias, doenças e gestão pública.

Percebeu-se que os Grupos Focais criaram um importante espaço para expressão dos desejos, percepções e angústias dos ACS, diante das vivências da capacitação de inclusão digital. A oportunidade dos grupos provocou uma espécie de catarse, onde tudo o que havia sido omitido nas respostas aos questionários pode ser expresso, e alguns sentimentos melhor compreendidos através do eco encontrado no grupo. De acordo com Caterall e Maclaran (1997) o grupo focal estimula a interação do grupo como algo produtivo que amplia o espectro de respostas, ativando detalhes de experiências esquecidas e desinibindo os participantes.

Através do espaço oferecido por meio do Grupo Focal para os capacitados foi dimensionando a importância da participação coletiva através de opiniões, sugestões e informações que poderão nortear projetos futuros. Nesse sentido, conclui-se pela necessidade de continuidade do processo através de projetos que estimulem a troca de experiências que focalizem a crítica sobre as práticas dos ACS, muitas vezes não exploradas e valorizadas devidamente.

A ideia de transformar a inclusão digital em Política Pública concretiza pressupostos como: o reconhecimento que a exclusão digital amplia a miséria e dificulta o desenvolvimento local e nacional, além de tornar fator de congelamento da condição de miserabilidade e de distanciamento entre as classes sociais; é a constatação de que o mercado não irá incluir na informatização os extratos pobres e desprovidos de 
dinheiro; por fim a aceitação de que a liberdade de expressão e o direito de se comunicar seria uma falácia se ele fosse apenas à minoria que tem acesso a comunicação em rede (SILVEIRA, 2005).

Nesse sentido, faz-se necessário uma (re) organização do Sistema Único de Saúde e assim uma re- definição do programa dos Agentes Comunitários de Saúde como instrumento e estratégia de articulado em diferentes níveis (estadual e municipal), onde os ACS possam ser qualificados, capacitados nos recursos multimídias e assim possam contribuir para o processo de saúde coletiva (POZ, 2002).

É importante assinalar que o projeto vislumbrou a ideia do "aprender para capacitar", como suficiente para disseminar a aprendizagem e manejo nos recursos multimídias. No entanto tal intuito não foi alcançado, uma vez que o índice de analfabetismo digital atingia a maioria dos capacitados, o que dificultou transformá-los em multiplicadores tecnológicos durante a capacitação.

Conclui-se que para que projetos como esse possam desenvolver plenamente seus objetivos é necessário ser assumido como algo pertencente aos sujeitos que dele irão fazer uso e que as peculiaridades e necessidades regionais sejam mote para sua reestruturação, a partir da negociação e preparação dos gestores municipais, que são fundamentais para o andamento do mesmo. Observou-se que o espaço oferecido por meio do Grupo Focal ampliou e aprofundou o conteúdo para análise, muitas vezes se revelando contraditório aos resultados dos questionários e formulários.

Nessa fase da pesquisa os sujeitos expressaram, além de suas opiniões e percepções, uma análise mais critica do que lhes costuma ser oferecido como capacitação, e que muitas vezes não é aproveitado pela falta de condições de diversas ordens como pessoais, institucionais e locais. Foi dimensionada a importância da participação coletiva, bem como a necessidade de continuidade do processo de troca de experiências 
e de grupos de reflexão sobre as práticas quotidianas dos usuários da capacitação, que muitas vezes não são valorizadas, através de projetos que envolvam intervenções grupais, a exemplo de grupos operativos.

\section{REFEREENCIAS}

BRASIL. Estratégias para a Inclusão Digital. Comitê executivo do governo eletrônico. Disponível em: <http:// www.governoeletronico.gov.br>. Acesso em: 15 ago. 2009.

BRASIL. MINISTÉRIO DA SAÚDE. Lei no 10.507, de 10 de julho de 2002. Cria a profissão de agente comunitário de saúde e dá outras providências. Brasília: Ministério da Saúde, 2002.

CATERALL, M.; MACLARAN, P. Focus group data and qualitative analysis programs: coding the moving picture as well as the snapshots. Sociological Research Online, v. 2, n.1, mar. 1997.

Comitê Gestor da Internet. 2006. Disponível em: <www.telecentros.desenvolvimento.gov.br>. Acesso em: 18 jan. 2011.

DIAS, C. A. Grupo focal: técnicas de coletas de dados em pesquisas qualitativas. (SD). Informação \& Sociedade: estudos, v. 10, n. 2, João Pessoa, 2000. Disponível em: <http://www.scielo.br>. Acesso em: 19 maio 2009.

HEIDMANN, I. et al. (Orgs.). Promoção à saúde: trajetória histórica de suas concepções. Texto contexto - enfermagem, vol.15 n. 2 , Florianópolis, 2006. Disponível em: <http://www.scielo.br>. Acesso em: 16 maio 2009.

Instituto Brasileiro de Geografia e Estatística. Censo Geográfico e Estatístico. Brasília, DF, 2000. Instituto Brasileiro de Opinião e Pesquisa. Disponível em: <http://www.ibope.com.br/calandraWeb/servlet/CalandraRedir

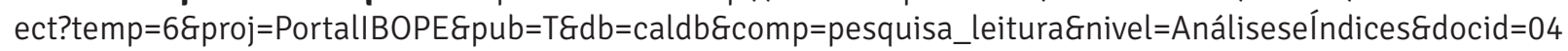
550BFD66F5DBEA83256EDD0074CDAA>. Acesso em: 12 set. 2010.

LÉVY, P. A conexão planetária. São Paulo: 34, 2003.

POZ. M. R. O agente comunitário de saúde: algumas reflexões. Interface - Comunicação, Saúde, Educação, v. 6, n. 10, p. 75-94, 2002.

SILVERIA, S. A. Inclusão Digital, software livre e globalização contra-hegemônica. Parceria Estratégicas, 2005.

VALENTE, A. et al. (Orgs.). Inclusão digital: tecendo redes afetivas / cognitivas. Prefácio. Rio de Janeiro: 2005. 
1 Psicóloga, Mestranda em Saúde e Ambiente pela Universidade Tiradentes - UNIT. Laboratório de Planejamento e Promoção de Saúde - LPPS. E-mail: e-mail: jamileteles18@hotmail.com.

2 Psicóloga (Unit) Aracaju/SE - Brasil. E-mail: psical@yahoo.com.br

3 Doutor em Ciências da Comunicação /USP, Professor do Programa de Pós-Graduação em Educação da Universidade Tiradentes. Pesquisador convidado do Núcleo de Estudos em Saúde Pública (NESP/UNB), Coordenador estadual do Programa ETEC Brasil. Aracaju/SE - Brasil. E-mail: ronaldo_linhares@unit.br

Doutora em Saúde Pública (UCPE), Professora do Mestrado em Saúde e Ambiente, Universidade Tiradentes/ Aracaju/SE, pesquisadora do LPPS-ITP. E-mail: criscunhaoliva@yahoo.com.br

5 Doutora em Psicologia Ciência e Profissão pela Pontifícia Universidade Católica de Campinas. Professora Titular do Departamento de Psicologia e do Programa de Pós-Graduação em Saúde e Ambiente da Universidade Aceito em: 23 de abril de 2013 Tiradentes, SE. E-mail: marlizete@uol.com.br 